\title{
Biomechanical parameters of the BP-enriched bone cement
}

\author{
Lukasz Matuszewski - Grażyna Olchowik - Tomasz Mazurkiewicz • \\ Bartłomiej Kowalczyk - Agata Zdrojewska - Anna Matuszewska • \\ Andrzej Ciszewski • Małgorzata Gospodarek · Iwona Morawik
}

Received: 4 April 2013/Accepted: 28 April 2013/Published online: 14 May 2013

(C) The Author(s) 2013. This article is published with open access at Springerlink.com

\begin{abstract}
Bisphosphonates (BPs) are well-known substances with very efficient antiresorptive properties. Their beneficial actions are useful not only in achieving better bone mineral density but also in improving bone microarchitecture, strength and, consequently, its quality. Surgical cement, being a polymer composite, is required to be highly biocompatible and biotolerant. The goal of the presented study was to assess whether the enrichment of cement with pamidronate has changed its biomechanical properties. We compared the biomechanical parameters of clean bone cement and BP-enriched bone cement, which were both used formerly in our rat models. Biomechanical properties of BP-enriched bone cement are defined by two basic terms: stress and strain, which are caused by the influence of external force. In the investigatory process of the bone's biomechanical parameters, the compressive test and the three-point flexural tests were used. During the
\end{abstract}

Ł. Matuszewski $(\bowtie) \cdot$ A. Ciszewski

Pediatric Orthopedic and Rehabilitation Clinic, Medical

University of Lublin, Ul. Chodźki 2, 21-093 Lublin, Poland

e-mail: lukasz.matuszewski@am.lublin.pl

G. Olchowik · B. Kowalczyk · A. Zdrojewska - M. Gospodarek Department of Biophysics, Medical University of Lublin, Lublin, Poland

T. Mazurkiewicz

Orthopedic and Traumatology Department, Medical University of Lublin, Lublin, Poland

\author{
A. Matuszewska \\ Biochemistry Department, Maria-Curie Sklodowska University \\ of Lublin, Lublin, Poland \\ I. Morawik \\ Orthopedic and Rehabilitation Department, Medical University \\ of Lublin, Lublin, Poland
}

three-point flexural investigation, the sample was supported at both ends and loaded in the middle, resulting in a flexure. After a specific range of flexure, the sample was fractured. In obtained results, there were no significant differences in the values of the stress determined at the point of maximal load and the energy stored in the samples for proportional stress-strain limit (elastic region). There were also no significant differences in the density of the samples. The study shows that the enrichment of bisphosphonates causes yielding of the bone cement material. In the presented data, we conclude that use of pamidronate implanted in bone cement did not have a detrimental effect on its biomechanical properties. Therefore, the obtained results encouraged us to perform further in vivo experiments which assess the biomechanical properties of bones implanted with BP-enriched bone cement.

Keywords Bisphosphonates - Bone cement . Biomechanics · Orthopedic

\section{Introduction}

Bisphosphonates (BPs) are well-known substances with very efficient antiresorptive properties [1,2]. Therefore, BPs have important effects on bone turnover and are widely used to treat a variety of diseases such as osteoporosis, bone metastasis, hypercalcemia of malignancy, Paget's disease, osteogenesis imperfecta or fibrous dysplasia of bone [3, 4]. Their beneficial actions not only aid in the achievement of better bone mineral density also are useful in the improvement in bone microarchitecture, strength and, consequently, its quality [5, 6]. BPs regulate osteoblastic functions such as proliferation and differentiation, prevent osteoblast apoptosis, modulate osteoblastic 
production of extracellular matrix proteins and regulate osteoblastic expression and secretion of various growth factors and cytokines [7-9]. Recent studies indicate that the most promising roles for the BPs are the prevention of bone collapse following osteonecrosis and the enhancement of implant fixation. Such combination therapies that have both bone antiresorptive and anabolic agents appear to be of great promise for other further orthopedic applications [3].

Modern generation of cementing techniques has really affected the survivorship of the use of orthopedic implants. Despite the optimization of cement preparation and proper initial cementing technique, the biomechanical properties of the bone cement used seem to be very important in preventing the further aseptic loosening of the implant [10]. Nowadays, PMMA-based bone cement (methyl polymethacrylate) seems to be most effective in total joint replacements implants. The first use of PMMA in a hip joint alloplasty was in the early 1960s when Charnley anchored the hip joint endoprosthesis inside the femur. Surgical cement, being a polymer composite, sets using an elemental bonding prosthesis with bone, and thus, it is required to be highly biocompatible and biotolerant. Many kinds of cements, either as pure polymer or with admixtures of different substances, are used. Application of fillers is of great significance to the mechanical properties of the PMMA. Specificity of the working conditions forces the necessity of transition of extremely complex loading resulting from human motor activity. Working environment of the bone cement is highly aggressive, which dramatically increases the aging rate and causes higher enucleating of the cement, weakening the bone-cement-implant system. This phenomenon can lead to endoprosthesis loosening and to the necessity of a reimplantation procedure [11]. The most important mechanical properties of PMMA cement are tensile, compressive, shear and fracture toughness. The variation in these properties is related to differences in composition, mixing methods, aging, temperature and viscosity during application. Such biomechanical parameters are mostly responsible for dynamic and static loads. PMMA is a very specific material of high modulus and low ductility [10]. Therefore, a high degree of stiffness of the bone cement is very important. The lack of this stiffness is responsible for the creep deformation, which is the tendency of a solid material to move slowly or deform permanently over the time under the influence of stresses [12]. However, creep deformation can be influenced by such factors as porosity, cement composition or molecular weight [13].

The goal of the presented study was to assess whether the enrichment of bone cement with pamidronate has changed its biomechanical properties. We compared the biomechanical parameters of clean bone cement and BPenriched bone cement which were both used in our former rat model.
Our previous animal studies demonstrated that local treatment with BPs can affect the level of bone turnover markers in blood serum [14]. We also proved that there are significant changes in the microarchitectural bone structure during the local use of pamidronate. Therefore, BP-enriched bone cement used in rat models had a positive effect on bone formation [15].

\section{Materials and methods}

\section{Reagents}

Bisphosphonate, pamidronate (Pamifos 60), was purchased from Vipharm (Warsaw, Poland). Bone cement was purchased from CMW 1, DePuy International Ltd, England. Research was performed on probes of BP-enriched bone cement where $40 \mathrm{~g}$ of cement was mixed with $60 \mathrm{mg}$ pamidronate by using a vacuum mixer. Then, it was compared with probes of clean bone cement.

\section{Biomechanical testing}

Biomechanical properties of the material are defined by two basic terms: stress and strain, which are caused by the influence of external force applied to a solid. In the investigatory process of the bone's biomechanical parameters, the compressive test and the three-point flexural tests were used. During the threepoint flexural investigation, the sample was supported at both ends and loaded in the middle, resulting in a flexure. After a specific range of flexure, the sample was fractured.

The three-point flexural test of bone cement and bisphosphonate-enriched bone cement was performed using a computerized testing machine, Instron 5965L1684 (Fig. 1). Samples in the form of cylinders of diameter $d_{0}=12.0 \mathrm{~mm}$ were placed in the holder (length of support span $L=2.0 \mathrm{~cm}$ ) and then exposed to a cross-load with $2 \mathrm{~mm} /$ min speed which caused the fracture, according to the method [16, 17]. Based on mechanical characteristics obtained during three-point flexural test, the load $(F)$ and stress (M) at the point of maximal load which causes cracking, and deflection $(l)$ and strain $(\delta)$ of the sample to this point were determined. Treating the sample as a rod of radius $6.0 \mathrm{~mm}$, transverse Young's modulus $\left(E_{\tau}\right)$ :

$E_{\tau}=\frac{F L^{3}}{12 \pi r^{4} l}$

The maximum energy $(W)$ absorbed by the sample before the fracture occurred was also determined. This energy is defined as the area under the curve of mechanical characteristics to the point of the maximal load. From load-deflection characteristics, the stiffness $\left(H_{\tau}\right)$ was determined as the slope of rectilinear region. 


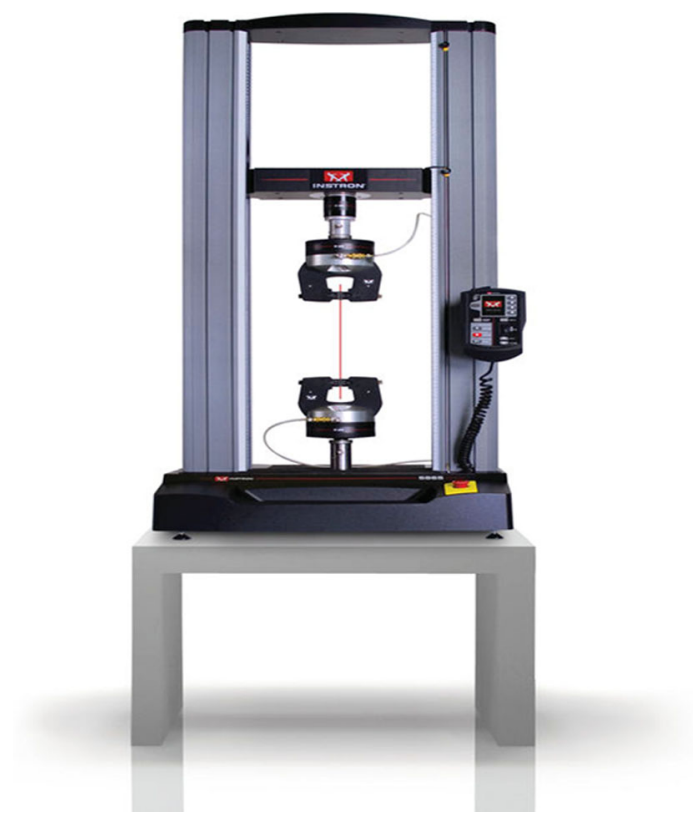

Fig. 1 Computerized testing machine Instron 5965L1684 was used in biomechanical testing of bone cement and bisphosphonate-enriched bone cement

Compression tests were performed on samples in the form of a cylinder with a diameter of $12.0 \mathrm{~mm}$ and height $10.0 \mathrm{~mm}$ using the same measuring device (Instron 5965L1684). Longitudinal Young's modulus $\left(E_{\mathrm{p}}\right)$ and stiffness $\left(H_{\mathrm{p}}\right)$ of the samples at maximum load $5 \mathrm{kN}$ were determined. In this regard, none of the samples had been destroyed.

Density of the samples was determined by using gas densitometer ULTRAPYC 1200e.

\section{FTIR measurements}

Fourier-transform infrared spectra were recorded with Nicolet 6700 FTIR spectrometer from Thermo Scientific Company. To register the FTIR spectrum of each sample, $1 \mathrm{mg}$ of powder of bone cement and $1 \mathrm{mg}$ of the powder of bisphosphonate-enriched bone cement were mixed with $200 \mathrm{mg} \mathrm{KBr}$ and compressed into a pellet for FTIR analysis. The spectra were received in the range of $400-3,000 \mathrm{~cm}^{-1}$ with a frequency resolution of $4 \mathrm{~cm}^{-1}$ in the transmission mode. For each sample, 16 scans were accumulated, Fourier-transformed and averaged. Background spectra were recorded before each sample to exclude the impact of external factors.

\section{Statistical analysis}

Statistical analyses were performed using one-way analyses of variance (ANOVA). Post-hoc comparison of means was carried out with Tukey's test for multiple comparisons, when appropriate. For this case, all data are shown as the mean \pm SEM. For cases with rejected hypothesis of normal distribution or homogeneity of variance, nonparametric ANOVA rank and Kruskal-Wallis multiple comparisons were used. The data are shown as the median with the first and third quartiles. The data were considered statistically significant at confidence limit of $p<0.05$. All statistical calculations were performed using 10.0 STATISTICA software (StatSoft, Poland).

\section{Results}

A typical load-deflection curve for bone cement samples is shown at Fig. 2 and for bisphosphonate-enriched bone cement at Fig. 3. The value of the load $(F)$ was registered on the ordinate axis and the deflection $(l)$ of the samples (the movement of the head shift) on the abscissa axis.

Biomechanical parameters were determined based on the characteristics from the three-point flexural test and the compressive test, and the densities $(\rho)$ of the bone cement and bisphosphonate-enriched bone cement samples are shown in Table 1. The table shows the mean values, standard deviation and the statistical significance level between bisphosphonate-enriched bone cement samples and pure bone cement samples.

The examinations conducted show that samples were destroyed in the elastic region. There were no significant differences of maximal load, but in the case of bisphosphonate-enriched bone cement, we observed an increase in strain $(4.5 \%)$ in comparison with pure bone cement. Stiffness of the bisphosphonate-enriched bone cement samples was $5 \%$ less during three-point flexural test $(p=0.0087)$ and about $1 \%$ during the compressive test $(p=0.06)$. Significant changes were observed in the values of transverse Young's modulus, which was about $4 \%$ less in bisphosphonate-enriched bone cement samples.

There were no significant differences in the values of the stress determined at the point of maximal load and the energy stored in the samples for proportional stress-strain limit (elastic region). There were also no significant differences in the density of the samples.

The study shows that the enrichment of bisphosphonates causes yielding of the bone cement material. Due to the yielding, the elastic region of the material is not permanently destroyed when the same mechanical load is increased.

FTIR spectra of the bone cement and the bisphosphonate-enriched bone cement are shown in Fig. 4. Enrichment of bone cement with BP did not cause the appearance of additional peaks, indicative of the formation of new interaction (chemical bonds). A low-boost spectrum (slightly more intensive) at 808, 1,145, 1,193, 1,243, 1,452, 
Fig. 2 Load-deflection curve for bone cement obtained during three-point flexural test
Fig. 3 Load-deflection curve for bisphosphonate-enriched bone cement obtained during three-point flexural test
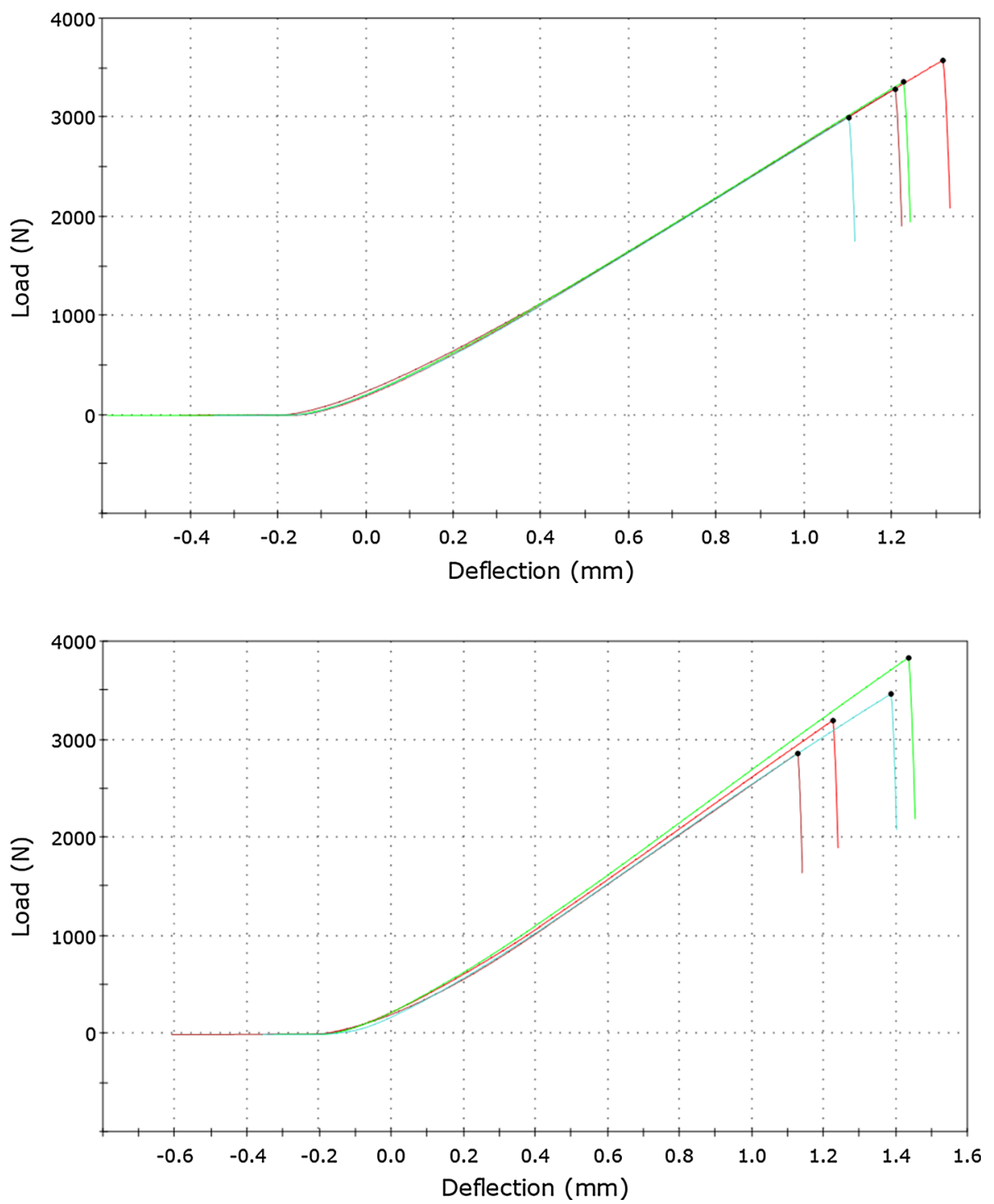

$1,487,1,523$ wavelengths was observed. This indicates that the enrichment of bone cement with bisphosphonate in ratio of $40 \mathrm{~g}$ of bone cement to $60 \mathrm{mg}$ of BP-enriched bone cement does not cause any visible changes in the chemical composite of bone cement.

\section{Discussion}

The purpose of this study was to investigate the biomechanical effects of enriching bone cement with pamidronate. Some researchers showed that enrichment of bone cement with antibiotics changed some of its biomechanical properties [18]. Others proved that even large amount of antibiotics implanted into bone cement did not adversely affect its mechanical properties [19]. Therefore, bone cement with antibiotics is widely used. Enrichment of bone cement with BP allows the regulation of important mediators involved in osteoclastogenesis, such as RANKL synthesized by osteoblasts that modulates OPG, a decoy receptor of RANKL-absorbing and preventing RANK activation. In our earlier report, we proved that use of the bisphosphonate that was present in the bone cement had a positive effect on bone turnover by acting directly on some important markers in the rats' serum. Our next micro-CT study showed other beneficial effects on the bone's microarchitecture [15]. Implanted BP-enriched bone cement simply changes the bone turnover in normal rats' bone toward a positive balance between bone formation and bone resorption. All those factors lead to a positive effect on bone formation together with enhanced growth.

In this paper, we used our own mathematical model for the calculations concerning the bone cement and the BPenriched bone cement's response to load; however, we obtained results similar to those presented by Lewis in the analysis of mechanical properties of PMMA bone cement 
[20]. We would also like to point out that there are major difficulties when comparing some of the results of the mechanical tests because of the variety of test conditions, sample preparation and methods of data analysis. However, we have to add that the stiffness of the bisphosphonateenriched bone cement in the compressive test and Young's modulus was significantly decreased but only less than $5 \%$. Therefore, most important mechanical properties of

Table 1 Biomechanical parameters and density of bone cement and bisphosphonate-enriched bone cement

\begin{tabular}{lccl}
\hline Parameter & $\begin{array}{l}\text { Bone } \\
\text { cement } \pm \mathrm{SD}\end{array}$ & $\begin{array}{l}\text { Bisphosphonate-enriched } \\
\text { bone cement } \pm \mathrm{SD}\end{array}$ & $p$ \\
\hline$F(\mathrm{~N})$ & $3,306 \pm 239$ & $3,324 \pm 437$ & 0.7232 \\
$M(\mathrm{MPa})$ & $95.00 \pm 6.86$ & $92.69 \pm 12.57$ & 0.7232 \\
$l(\mathrm{~mm})$ & $1.21 \pm 0.09$ & $1.24 \pm 0.17$ & 0.7363 \\
$\delta(\%)$ & $22.0 \pm 2.0$ & $24.0 \pm 3.0$ & $>0.8$ \\
$E_{\tau}(\mathrm{MPa})$ & $433.78 \pm 1.71$ & $415.03 \pm 12.04$ & 0.0087 \\
$H_{\tau}(\mathrm{N} / \mathrm{mm})$ & $2,773.3 \pm 9.8$ & $2,607.3 \pm 88.4$ & $0.0132^{*}$ \\
$W(\mathrm{~J})$ & $2.07 \pm 0.29$ & $2.13 \pm 0.51$ & $>0.8$ \\
$E_{\mathrm{p}}(\mathrm{MPa})$ & $1,240.0 \pm 7.0$ & $1,229.0 \pm 10.0$ & 0.1194 \\
$H_{\mathrm{p}}(\mathrm{kN} / \mathrm{mm})$ & $14.23 \pm 0.06$ & $14.10 \pm 0.11$ & 0.0656 \\
$\rho\left(\mathrm{kg} / \mathrm{m}^{3}\right)$ & $1,248 \pm 10$ & $1,250 \pm 10$ & 0.7599 \\
\hline
\end{tabular}

$F$-load, $M$-stress, $l$-deflection, $\delta$-strain, $E_{\tau}$-transverse Young's modulus (three-point flexural test), $H_{\tau}$-stiffness from the three-point flexural test, $W$ - energy stored in the sample before fracture, $E_{\mathrm{p}}$-longitudinal Young's modulus (compressive test), $H_{\mathrm{p}}$-stiffness from compressive test, $\rho$-density

* Significance value
BP-enriched cement were preserved [20]. Other physical and mechanical properties such as density and maximal load were the same or even increased as in the case of strain. Implantation of pamidronate into the bone cement did not change chemical properties also.

Some researchers showed that the stress-strain limit of bone cement might be associated with prosthetic loosening [21]. Yielding is a response to the plastic deformation of bone cement, but when it reaches levels over the yield stress level, it causes permanent deformation. Such deformation, also known as creep deformation, is undesirable in bone cement [12]. In our experimental model, enrichment of bisphosphonates caused yielding of the bone cement material. It may even improve its efficiency during mechanical loading tests. However, it needs some further in vivo studies and analysis concerning fatigue potential of BP-enriched cement.

It is a well-known fact that bisphosphonates inhibit osteoclast-mediated bone resorption, play an important role in the healing of fractured bones, reduce osteoarthritis, prevent prosthetic migration or enhance bone allograft ingrowth [22-25]. Despite the fact that the effects of bisphosphonates on material-level properties of bone have been evaluated by many authors [26-28], the biomechanical properties of BP-enriched bone cement have not been investigated so far. In the presented data, we conclude that the implantation of pamidronate into bone cement did not aggravate its biomechanical properties. We believe that the local use of BP-enriched bone cement may be very efficient
Fig. 4 FTIR spectra of the bone cement and the BP-enriched cement

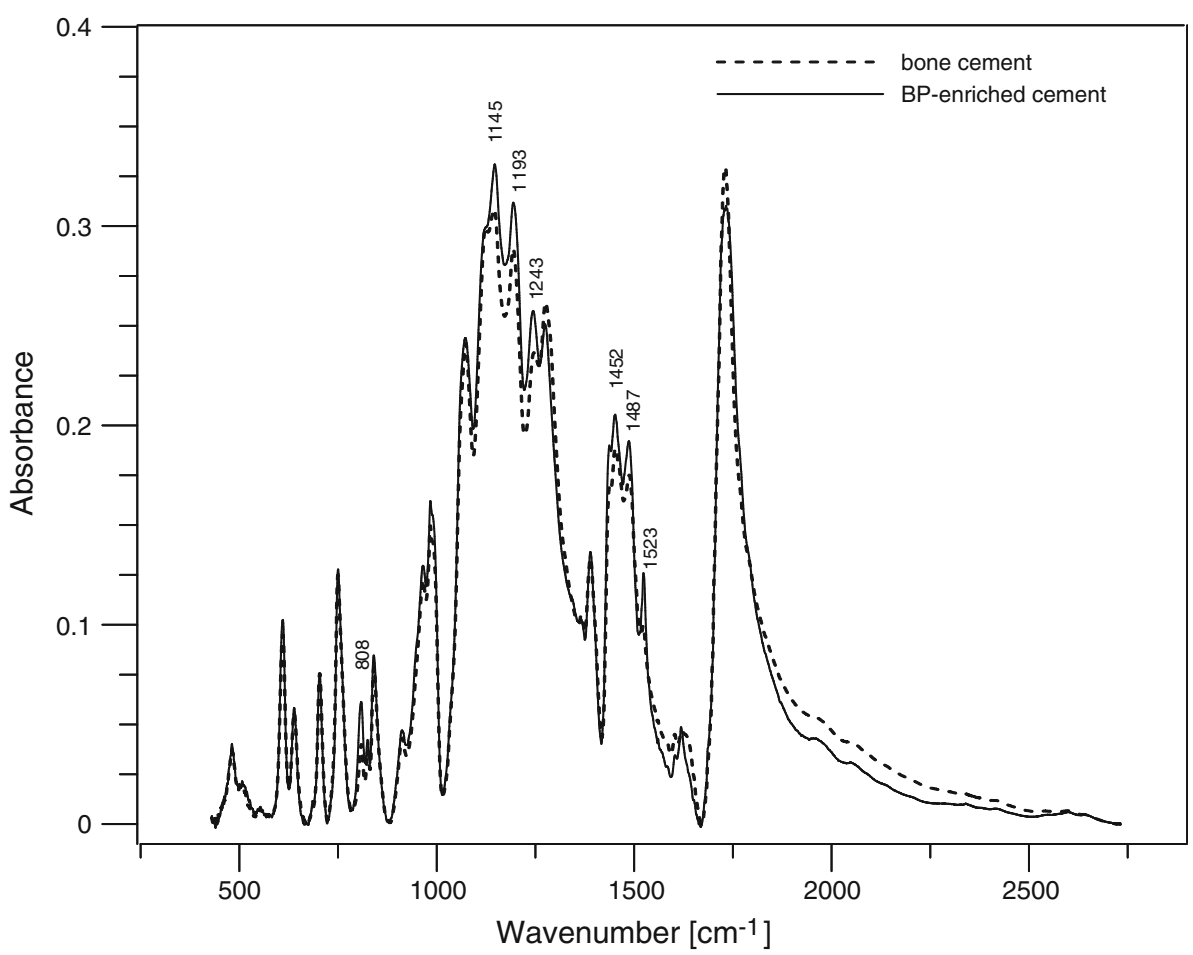


in the treatment of various bone diseases in humans, especially in those who require a positive balance between bone formation and bone resorption; therefore, the concept of using BP-enriched bone cement implants appears to be justified. We would like to add that BP-enriched bone cement has only been used in animal models thus far, but the results that we have obtained have encouraged us to perform additional in vivo experiments that assess the biomechanical properties of bones implanted with BPenriched bone cement. Those experiments together with former research enable us to start new scientific programs which facilitate the potential use of BP-enriched bone cement and its further clinical application.

Conflict of interest No funds were received in support for this study. The authors of this manuscript have chosen not to furnish EJOST and its readers with information regarding any relationship that might exist between a commercial party and material contained in this manuscript that might represent a potential conflict of interest. No benefits in any form have been or will be received from a commercial party related directly or indirectly to the subject of this manuscript. Although the authors have not received and will not receive benefits for personal or professional use from a commercial party related directly or indirectly to the subject of this manuscript, benefits have been or will be received but are directed solely to a research fund, foundation, educational institution or other non-profit organization with which one or more of the authors are associated. The authors have received or will receive benefits for personal or professional use from a commercial party related directly or indirectly to the subject of this manuscript. These benefits have been or will be directed to a research fund, foundation, educational institution or other non-profit organization with which one or more of the authors are associated.

Open Access This article is distributed under the terms of the Creative Commons Attribution License which permits any use, distribution, and reproduction in any medium, provided the original author(s) and the source are credited.

\section{References}

1. Fleisch H (1998) Bisphosphonates: mechanisms of action. Endcr Rev 19:80-100

2. Russell RGG, Rogers MJ (1999) Bisphosphonates: from the laboratory to the clinic and back again. Bone 25:97-106

3. Wilkinson JM, Little DG (2011) Bisphosphonates in orthopedic applications. Bone 49:95-102

4. Mundy GR, Yoneda T (1998) Bisphosphonates as anticancer drugs. N Engl J Med 339:398-400

5. Watts NB, Harris ST, Genant HK, Wasnich RD, Miller PD, Jackson RD, Licata AA, Ross P, Woodson GC III, Yanover MJ, Mysiw WJ, Kohse L, Rao MB, Steiger P, Richmond B, Chesnut CH III (1990) Intermittent cyclical etidronate treatment of postmenopausal osteoporosis. N Engl J Med 323:73-79

6. Saag KG, Emkey K, Schnitzer TJ, Brown JP, Hawkins F, Goemaere S, Thamsborg G, Liberman UA (1998) Alendronate for the prevention and treatment of glucocorticoid-induced osteoporosis. Glucocorticoid-induced osteoporosis intervention study group. N Engl J Med 339:292-299
7. Reinholz GG, Getz B, Pederson L, Sanders ES, Subramaniam M, Ingle JN, Spelsberg TC (2000) Bisphosphonates directly regulate cell proliferation, differentiation, and gene expression in human osteoblasts. Cancer Res 60:6001-6007

8. Giuliani N, Pedrazzoni M, Negri G, Passeri G, Impicciatore M, Girasole G (1998) Bisphosphonates stimulate formation of osteoblast precursors and mineralized nodules in murine and human bone marrow cultures in vitro and promote early osteoblastogenesis in young and aged mice in vivo. Bone 22:455-461

9. Åstrand J, Aspenberg P (2002) Reduction of instability-induced bone resorption using bisphosphonates. High doses are needed in rats. Acta Orthop Scand 73(1):24-30

10. Callaghan J, Aaron G, Rosenberg MD, Rubash HE (2007) The adult hip. Lippincott Williams \& Wilkins, Philadelphia

11. Włodarski J, Szyprowski J, Więckowski W, Szarek A (2005) Fillers influence on the mechanical properties of the composite bone cements. Composites 5(4):78-81

12. Verdonschot N, Huiskes R (1994) Creep behavior of hand-mixed Simplex $\mathrm{P}$ bone cement under cyclic tensile loading. J Appl Biomater 5:235-243

13. Orr JF, Dunne NJ, Quinn JC (2003) Shrinkage stresses in bone cement. Biomaterials 24:2933-2940

14. Mazurkiewicz T, Matuszewski Ł, Matuszewska A, Jaszek M (2013) Implanted bisphosphonates in bone cement affect bone markers in rat serum. Int Orthop. Online: http://link.springer.com/ article/10.1007/s00264-013-1816-6. Accessed 26 Jan 2013

15. Matuszewski Ł, Turżańska K, Matuszewska A, Jabłoński M, Polkowska I, Mazurkiewicz T (2013) Effect of implanted bisphosphonate-enriched cement on the trabecular microarchitecture of bone in a rat model using micro-computed tomography. Int Orthop. Online: http://www.springer.com/alert/urltracking.do? id=Lfb8d2bMbe6f65Saad8ca1. Accessed 24 Feb 2013

16. Kaneps AJ, Stover SM, Lane NE (1997) Changes in canine cortical and cancellous bone mechanical properties following immobilization and remobilization with exercise. Bone 21(5): 419-423

17. Olchowik G, Chadaj-Polberg E, Tomaszewski M, Polberg M, Tomaszewska M (2011) The influence of caffeine on the biomechanical properties of bone tissue during pregnancy in a population of rats. Folia Histochem Cytobiol 49(3):504-511

18. Buchholz HW, Engelbrecht H (1970) Uber die depotvirkung einiger antibiotic bei vermiscgung dem kunstharz Palcos. Chirurg 41:511-515

19. Davies JP, O'Connor DO, Burke DW et al (1989) Influence of antibiotic impregnation on the fatigue life of Simplex $\mathrm{P}$ and Palacos R acrylic bone cements, wit and without centrifugation. J Biomed Mater Res 23:379-397

20. Lewis G (2003) Fatigue testing and performance of acrylic bone cement materials: state-of-the-art review. J Biomed Mater Res B Appl Biomater 66:457-486

21. Topoleski LD, Ducheyne P, Cuckler JM (1995) The effects of centrifugation and titanium fiber reinforcements on fatigue failure mechanisms in poly(methyl methacrylate) bone cement. Biomed Mater Res 28:299-307

22. Jones MD, Tran CW, Li G, Maksymowych WP, Zernicke RF, Doschak MR (2010) In vivo microfocal computed tomography and micro-magnetic resonance imaging evaluation of antiresorptive and antiinflammatory drugs as preventive treatments of osteoarthritis in the rat. Arthr Rheum 62(9):2726-2735

23. Allen MR (2011) The effects of bisphosphonates on jaw bone remodeling, tissue properties, and extraction healing. Odontology 99(1):8-17

24. Schindeler A, Birke O, Yu NY, Morse A, Ruys A, Baldock PA, Little DG (2011) Distal tibial fracture repair in a neurofibromatosis type 1-deficient mouse treated with recombinant bone 
morphogenetic protein and a bisphosphonate. J Bone Jt Surg Br 93(8):1134-1139

25. Qi M, Hu J, Li J, Li J, Dong W, Feng X, Yu J (2012) Effect of zoledronate acid treatment on osseointegration and fixation of implants in autologous iliac bone grafts in ovariectomized rabbits. Bone 50(1):119-127

26. Hilding M, Ryd L, Toksvig-Larsen S, Aspenberg P (2000) Clodronate prevents prosthetic migration: a randomized radiostereometric study of 50 total knee patients. Acta Orthop Scand 71(6):553557

27. Aspenberg A, Astrand J (2002) Bone allograft pretreated with bisphosphonate are not resorbed. Acta Ortop Scand 73(1):20-23

28. Catterall JB, Cawston TE (2003) Drugs in development: bisphosphonates and metalloproteinase inhibitors. Arthr Res Ther $5: 12-24$ 